\title{
NUMERICAL ANALYSIS OF COMBUSTION FOR DROPLET OF HYDRAZINE
}

\author{
M. Motoe \\ The Japan Aerospace Exploration Agency \\ 3-1-1 Yoshinodai, Chuuou, Sagamihara, Kanagawa-ken 252-2510, Japan
}

\begin{abstract}
Several simulations of evaporation and combustion of a fuel droplet are performed as preliminary stage for the combustion simulation in the chamber of bipropellant thrusters. First, the evaporation simulation without chemical reaction for single droplets of water and $n$-heptane are performed. Two evaporation models such as classical equilibrium and nonequilibrium Langmuir-Knudsen models are used for the simulation, and their characteristics are studied in order to investigate the difference between the two models. Then, the accuracy of the numerical code using this study is confirmed by means of comparison with existing experimental results. Next, the evaporation simulation with chemical reaction for single droplets of hydrazine is performed and the accuracy of the numerical code is confirmed by the same way. From these simulations, it is considered that the code and the scheme using this simulation have reasonable accuracy for droplet simulation with evaporation and combustion.
\end{abstract}

\section{INTRODUCTION}

Bipropellant thruster is a major propulsion system of spacecraft such as satellites and space probes. The propellants used for the thruster ignite when they mix each other. When these propellants are injected individually and are mixed in the combustion chamber of the thruster appropriately, they will burn without any artificial ignition. Therefore, the typical bipropellant thruster does not equip an igniter. A combination of hydrazine and dinitrogen tetraoxide or monomethyl hydrazine and dinitrogen, the former is fuel and the latter is oxidizer, are usually used as propellant of the thruster. Since both are in liquid phase, the fuel and the oxidizer are vaporized by atomization and then mixed. The atomization is conducted by means of impingement of the fuel and oxidizer flows. The flows

(C) The Authors, published by EDP Sciences. This is an open access article distributed under the terms of the Creative Commons Attribution License 4.0 (http://creativecommons.org/licenses/by/4.0/). 
form a liquid film. Then, the film becomes fragments from the edge of it by means of instability. Basically, the fragments are vaporized and ignite after mixing. Part of the propellant ignites in liquid phase [1].

Because the bipropellant thruster does not equip any igniter, it has merits as simple structure and lightweight. Not using any active ignition means that the timing of the ignition mainly depends on the conditions in the chamber, a mixing state of the propellant, and so on, and it is difficult to be predicted. Additionally, in the property of the spacecraft mounting the thruster, there are problems that are caused by pulse ignition at operations such as a combustion instability, ignition shock, and thermal load to the wall of the chamber. These various problems have so far prevented the appropriate design of the bipropellant thrusters. As a consequence, the designs have been conducted by using several combustion experiments and empirical rules.

In order to improve such situations, the numerical fluid simulation has been applied to predict the thermal load to the chamber wall and to investigate the chamber inner state $[2,3]$.

As baseline study of an analysis in the chamber of bipropellant thruster, the object of this study is to determine the combustion properties of the hydrazine droplet in ambient of oxidizer for various cases by the combustion simulation with detailed chemical reaction model of Daimon et al. [2]. This study is expected to give valuable knowledge in order to investigate the whole region of the bipropellant thrusters.

\section{GOVERNING EQUATIONS}

\subsection{Gaseous Phase}

In this study, the gaseous phase is described by the Eulerian method and the droplets are described by the Lagrangian method. Dominant equations of the gaseous phase, which are partially discretized at source term, are shown below:

$$
\begin{gathered}
\frac{\partial \rho}{\partial t}+\frac{\partial u_{i}}{\partial x_{i}}=\frac{1}{V} \sum_{k=1}^{n_{d}} \dot{m}_{d_{K}} \\
\frac{\partial \rho u_{i}}{\partial t}+\frac{\partial \rho u_{i} u_{j}}{\partial x_{j}}=-\frac{\partial p}{\partial x_{i}}+\frac{\partial \tau_{i j}}{\partial x_{j}}+\frac{1}{V} \sum_{k=1}^{n_{d}} F_{d_{i_{k}}} \\
\frac{\partial \rho e}{\partial t}+\frac{\partial \rho u_{j} H}{\partial x_{j}}=\frac{\partial}{\partial x_{j}}\left(u_{i} \tau_{i j}-q_{j}\right)+\frac{1}{V} \sum_{k=1}^{n_{d}} q_{d_{i_{k}}}
\end{gathered}
$$




$$
\begin{aligned}
\frac{\partial \rho Y_{n}}{\partial t}+\frac{\partial \rho u_{j} Y_{n}}{\partial x_{j}} & =\frac{\partial}{\partial x_{j}}\left(\rho D_{n} \frac{\partial Y_{n}}{\partial x_{j}}\right)+\frac{1}{V} \sum_{k=1}^{n_{p}} \dot{m}_{d_{n_{k}}} \\
\tau_{i j} & =\mu\left(\frac{\partial u_{j}}{\partial x_{i}}+\frac{\partial u_{i}}{\partial x_{j}}-\frac{2}{3} \frac{\partial u_{k}}{\partial x_{k}} \delta_{i j}\right) ; \\
q_{j} & =-\kappa \frac{\partial T}{\partial x_{j}} .
\end{aligned}
$$

In Eqs. (1) to (6), $t, x_{i}, \rho, u_{i}, p, e, H, T, Y_{n}, \tau_{i j}, q_{i}, \mu, \kappa, D_{n}$, and $\delta_{i j}$ are the time, Cartesian coordinate, density, velocity, pressure, total energy per unit mass, total enthalpy, temperature, mass fraction of chemical species, viscous stress tensor, heat flux, viscous coefficient, thermal conductivity, diffusion coefficient of a chemical species, and Kronecker delta, respectively. The " $i, j, k$ " are the index of tensor; " $n$ " is the index of chemical species; and " $n_{d}$ " is the droplet number included in a cell. Additionally, $\dot{m}_{d_{k}}, F_{d_{i_{k}}}$, and $q_{d_{i_{k}}}$ are the influence of mass, momentum, and energy from droplets in a cell, respectively.

\subsection{Movement of the Droplet}

The movement of the droplets is calculated by means of PSI-Cell (particle-sourcein cell) method [4] and the dominant equations of a single droplet are shown below:

$$
\begin{aligned}
\frac{d U_{d_{i}}}{d t} & =F_{d_{i}} \\
F_{d_{i}} & =\frac{\pi}{8} C_{D} \rho d_{d}^{2}\left|u_{i}-U_{p_{i}}\right|\left(u_{i}-U_{d_{i}}\right) ; \\
C_{D} & =\frac{24}{\operatorname{Re}_{d}}\left(1+0.15 \operatorname{Re}_{d}^{0.687}\right) ; \\
\operatorname{Re}_{d} & =\frac{\rho d_{d} \sqrt{\left(u_{i}-U_{d_{i}}\right)^{2}}}{\mu}
\end{aligned}
$$

Here, $U_{d_{i}}, C_{D}, d_{D}$, and $\operatorname{Re}_{d}$ are the velocity of droplet, drag coefficient of droplet, diameter of droplet, and Reynolds number based on relative velocity of gas and droplet and the diameter, respectively. 


\subsection{Evaporation of the Droplet}

In this study, the classic model [5] and the nonequilibrium model LangmuirKnudsen $[6,7]$ are performed for the evaporation of a droplet. Formulations of the classic model are shown below:

$$
\begin{aligned}
\frac{d m_{d}}{d t} & =-A_{d} N_{\mathrm{sd}} \\
N_{\mathrm{sd}} & =k_{c}\left(C_{\mathrm{sd}, s}-C_{\mathrm{sd}, \infty}\right) ; \\
k_{c} & =\frac{D_{\mathrm{sd}, m}}{d_{d}}\left(2.0+0.6 \mathrm{Re}_{d}^{1 / 2} \mathrm{Sc}^{1 / 2}\right) ; \\
C_{\mathrm{sd}, \infty} & =X_{\mathrm{sd}} \frac{p_{\infty}}{R_{0} T_{\infty}} \mathrm{MW}_{\mathrm{sd}} ; \\
C_{\mathrm{sd}, s} & =\frac{P_{\mathrm{sat}}}{R_{0} T_{d}} \mathrm{MW}_{\mathrm{sd}} ; \\
P_{\mathrm{sat}} & =P_{0} \exp \left\{\frac{L}{R_{0} / \mathrm{MW}_{\mathrm{sd}}}\left(\frac{1}{T_{0}}-\frac{1}{T_{d}}\right)\right\} ; \\
L & =\ln \left(\frac{P_{1}}{P_{0}}\right) \frac{R_{0} / \mathrm{MW}_{\mathrm{sd}}}{1 / T_{0}-1 / T_{1}} .
\end{aligned}
$$

Here, $m_{d}, A_{d}, N_{\mathrm{sd}}, k_{c}, D_{\mathrm{sd}, m}, \mathrm{Sc}, C_{\mathrm{sd}, s}, C_{\mathrm{sd}, \infty}, X_{\mathrm{sd}}, p_{\infty}, T_{\infty}, R_{0}, \mathrm{MW}_{\mathrm{sd}}, P_{\mathrm{sat}}$, $T_{d}$, and $L$ are the mass of droplet, surface area of droplet, vapor mass flux of droplet, mass transfer coefficient, mass diffusion coefficient between mixture gas and vapor of droplet, Schmidt number, vapor concentration at droplet surface, vapor concentration at mixture gas, mole fraction of species of vapor, pressure of mixture gas, temperature of mixture gas, universal gas constant, molecular weight of droplet component, saturated vapor pressure, droplet temperature, and latent heat, respectively; $P_{0}, T_{0}, P_{1}$, and $T_{1}$ are the reference pressure and temperature; and index "sd" means the component of chemical species of droplet.

Formulations of the nonequilibrium model are shown below:

$$
\begin{aligned}
\frac{d m_{d}}{d t} & =-\frac{S h}{3 \mathrm{Sc}} \frac{m_{d}}{\tau_{d}} H_{M} ; \\
\tau_{d} & =\frac{\rho_{d} d_{d}^{2}}{18 \mu} ;
\end{aligned}
$$




$$
\begin{aligned}
H_{M} & =\ln \left[1+B_{M, \text { neq }}\right] ; \\
B_{M, \text { neq }} & =\frac{Y_{s, \text { neq }}-Y_{\text {sd }, \infty}}{1-Y_{s, \text { neq }}} ; \\
Y_{s, \text { neq }} & =\frac{X_{s, \text { neq }}}{X_{s, \text { neq }}+\left(1-X_{s, \text { neq }}\right) \mathrm{MW}_{\text {mix }} / \mathrm{MW}_{\text {sd }}} ; \\
X_{s, \text { neq }} & =\frac{P_{\text {sat }}}{p_{\infty}}-\left(\frac{L_{K}}{d_{d} / 2}\right) \beta ; \\
L_{K} & =\frac{\mu \sqrt{2 \pi T_{d} R_{0} \mathrm{MW}_{\mathrm{sd}}}}{\operatorname{Sc} p_{\infty}} ; \\
\beta & =-\left(\frac{3 \operatorname{Pr} \tau_{d}}{2}\right) \frac{\dot{m}_{d}}{m_{d}} .
\end{aligned}
$$

Here, $\tau_{d}, H_{M}, \rho_{d}, B_{M \text {,neq }}, Y_{s, \text { neq }}, Y_{\text {sd }, \infty}, X_{s, \text { neq }}, \mathrm{MW}_{\text {mix }}, L_{K}$, and $\beta$ are the droplet time constant, specific driving potential for mass transfer, density of droplet, nonequilibrium Spalding transfer number for mass, nonequilibrium vapor surface mass fraction, mass fraction of species of vapor in mixture gas, nonequilibrium vapor surface mole fraction, molecular weight of mixture gas, Knudsen layer thickness, and nondimensional evaporation parameter, respectively.

\subsection{Heat Transfer of the Droplet}

A formulation of heat tranfer between droplet and gas which are performed with the classical model are shown below:

$$
m_{d} C_{L} \frac{d T_{d}}{d t}=h A_{p}\left(T_{\infty}-T_{d}\right)+L \frac{d m_{d}}{d t}
$$

In Eq. (9), $C_{L}$ and $h$ are the specific heat of droplet and heat transfer coefficients between droplet and mixture gas, respectively.

A formulation of heat tranfer which is performed with the nonequilibrium model is shown below:

$$
\frac{d T_{d}}{d t}=\frac{\mathrm{Nu}}{3 \operatorname{Pr}} \frac{\beta}{e^{\beta}-1} \frac{C_{p} / C_{L}}{\tau_{d}}\left(T_{\infty}-T_{d}\right)+\left(\frac{L_{V}}{C_{L}}\right) \frac{\dot{m}_{d}}{m_{d}} .
$$

In Eq. (10), $\mathrm{Nu}$ and $C_{p}$ are the Nusselt number and specific heat of mixture gas, respectively. 


\subsection{Chemical Reactions of Hydrazine}

Chemical reaction of hydrazine is calculated by means of the detailed chemical reaction model of Daimon et al. [2]. This model has 39 chemical species and 26 reactions.

\section{NUMERICAL METHODS}

The numerical code employed here is "LS-Flow:" JAXA's in-house, unstructured finite-volume method, compressible Navier-Stokes solver for arbitrary polygons/ polyhedral [8]. The numerical flux is calculated by means of SLAU (simple low-dissipation AUSM (advection upstream splitting method)) scheme of Shima and Kitamura [9], and the values of interface are interpolated by the 2ndorder MUSCL (monotone upstreamn scheme for conservation laws) scheme with Venkatakrishnan limiter. The time integration of gaseous phase is performed by means of the 2nd-order LU-SGS (lower-upper symmetric Gauss-Seidel) scheme with inner iteration. The time integration of chemical reaction is separated from that of gaseous phase and is performed by means of ERENA method of Morii et al. [10] which is based on an explicit time integration scheme.

\section{ANALYSIS OBJECT}

As a preliminary analysis, two examinations of evaporation of single droplet which one is water and the other is $n$-heptane are simulated in order to vali-

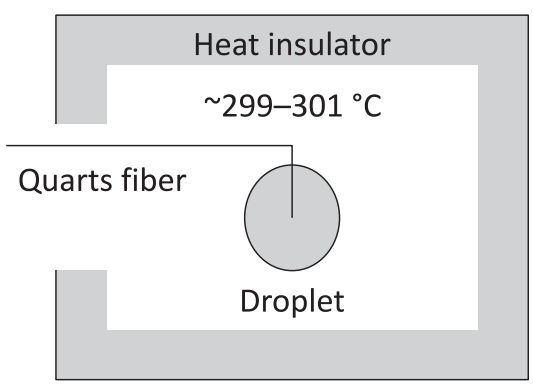

(a)

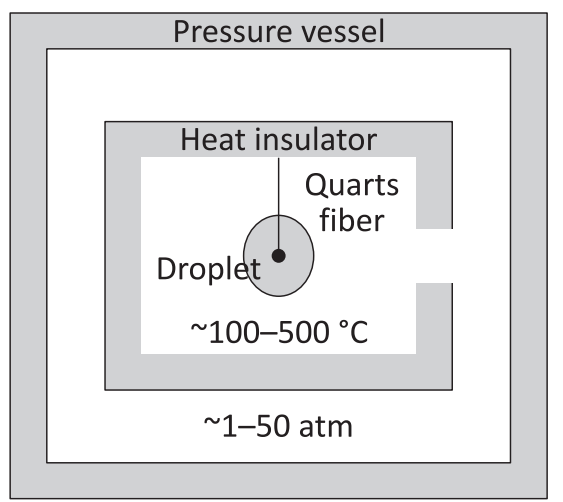

(b)

Figure 1 The examination of water $(a)$ and $n$-heptane $(b)$ droplets 
date the numerical code. The examination of water droplet was performed by Kobayashi [11]. A single droplet is suspended by slight fiber of quarts in the oven filled by high-temperature static air as shown in Fig. $1 a$, and the time series of diameters of the droplet are measured. The examination of $n$-heptane droplet was performed by Hiroyasu et al. [12]. The droplet is suspended by slight quarts fiber with spherical point in the pressure vessel which is filled by nitrogen as shown in Fig. $1 b$, and the temperature and the pressure in the chamber can be varied. Similarly to the examination of water, the time series of diameters of the droplet are measured.

Subsequently, the simulation of hydrazine droplet with chemical reaction is performed and it is based on an examination of Allison [13]. As shown in Fig. 2, in the simulation, a hydrazine droplet is

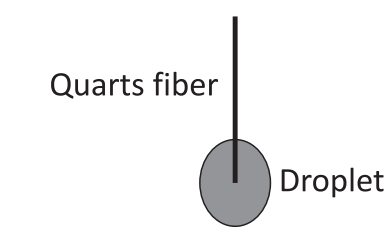

$\sim 53.4-63.8 \mathrm{~cm} / \mathrm{s} \sim 1660-2530 \mathrm{~K}$

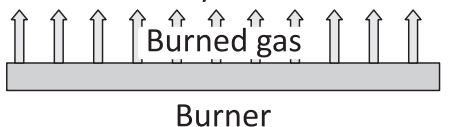

Burner

Figure 2 The examination of hydrazine droplet suspended by quarts fiber in burned gas

flow with high temperature, and the hydrazine will cause autolysis reaction. Time series of the droplet diameters are measured, and the mass burning rates $\dot{M}$ are calculated by means of

$$
\dot{M}=\frac{1}{4} \pi K \rho_{d} d_{d}
$$

using the gradient of the time series $K$ :

$$
K=\frac{d d_{d}^{2}}{d s}
$$

In this study, the simulations are performed for several cases of above examinations as shown in Table 1, and the initial droplet temperatures are arbitrarily fixed at $300 \mathrm{~K}$ because the references do not mention anything about it.

Table 1 The simulation condition

\begin{tabular}{lccccc}
\hline Droplet & $\begin{array}{c}\text { Initial droplet } \\
\text { diameter, } \\
\mathrm{mm}\end{array}$ & $\begin{array}{c}\text { Initial droplet } \\
\text { temperature, }\end{array}$ & $\begin{array}{c}\text { Ambient } \\
\text { temperature, }\end{array}$ & $\begin{array}{c}\text { Ambient } \\
\text { pressure, } \\
\text { atm }\end{array}$ & $\begin{array}{c}\text { Free stream } \\
\text { velocity, } \\
\mathrm{m} / \mathrm{s}\end{array}$ \\
\hline Water & 1.38 & 300 & 572 & 1 & 0 \\
$n$-heptane & 1.8 & 300 & 773 & 1 & 0 \\
Hydrazine & 1.3 & 300 & 2060 & 1 & 0.05576 \\
\hline
\end{tabular}




\section{NUMERICAL SETUP}

Figure $3 a$ shows the numerical grid used for the simulations of water and $n$-heptane droplet evaporation. As shown in the figure, the grid is a 0.704-meter cube which is divided in 11 equal intervals on a side; therefore, the number of cells is 1331 and the grid space is $0.064 \mathrm{~m}$. The droplet is located at the center of the grid and quarts fiber and other equipment are not concerned. The time step of calculation for water droplet is $2 \mu \mathrm{s}$ and for $n$-heptane droplet is $1 \mu \mathrm{s}$. The boundary condition that pressure and temperature are fixed at the ambient values as shown in Table 1 is common in all boundaries. These simulations are performed by a workstation which has two Intel ${ }^{\circledR}$ Xeon ${ }^{\circledR}$ E5-2640 6-core CPU (central processing unit) divided into 12 areas and parallelized by MPI (message passing interface).

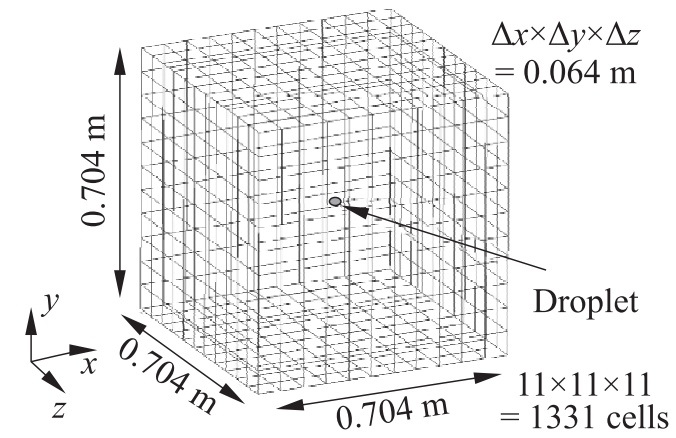

(a)

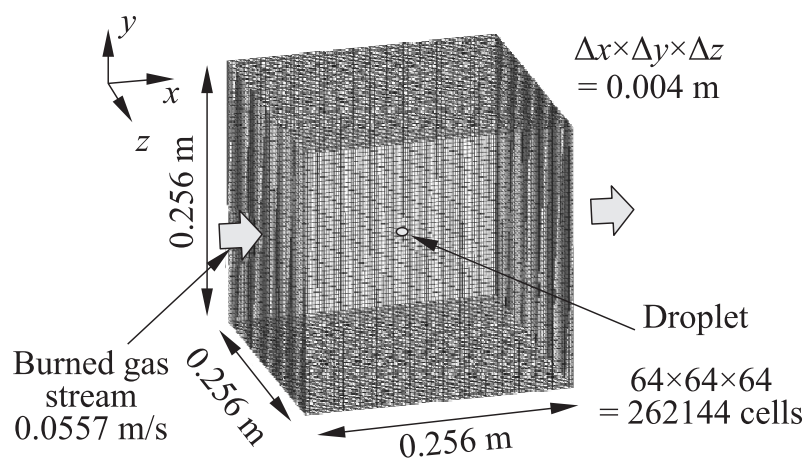

(b)

Figure 3 Numerical grids for water and $n$-heptane $(a)$ and for hydrazine $(b)$ droplets 
Figure $3 b$ shows the numerical grid used for the simulations of hydrazine droplet evaporation. As shown in Fig. $3 b$, the grid is a 0.256 -meter cube which is divided in 64 equal intervals on a side. The number of cells is 262144 and the grid space is $0.004 \mathrm{~m}$. The droplet is located at the center of the grid and quarts fiber and other equipment are not modeled similarly above. The time step of this calculation is $0.2 \mu \mathrm{s}$. The free stream flows to forward $x$ direction with the speed of $0.0557 \mathrm{~m} / \mathrm{s}$, the pressure of $1 \mathrm{~atm}$, and the temperature of $2060 \mathrm{~K}$. The same as in the former simulations, the boundary condition that velocity, pressure, and temperature are fixed in the free stream condition is common in all boundary. The simulations are performed on JAXA Supercomputer System generation 2 (JSS2) divided into 995 areas and parallelized by MPI.

\section{RESULTS}

The results of evaporation simulations of water and $n$-heptane are shown in Fig. 4. The calculations are performed by using two evaporation models mentioned above. From Fig. 4a, though it can be seen that differences of evaporation time between the two numerical results and the experimental result are about $10 \%$ of the experimental, the slopes are well corresponding to the experimental. It can be thought that the initial droplet temperature has a lag between the experimental and it causes the difference of the evaporation time. In this case, the two evaporation models indicate roughly the same results. The result of nonequilibrium is longer than the equilibrium one. It is because the calculated mole fraction of evaporated droplet is smaller than the one at equilibrium condition as shown in Eq. (7).

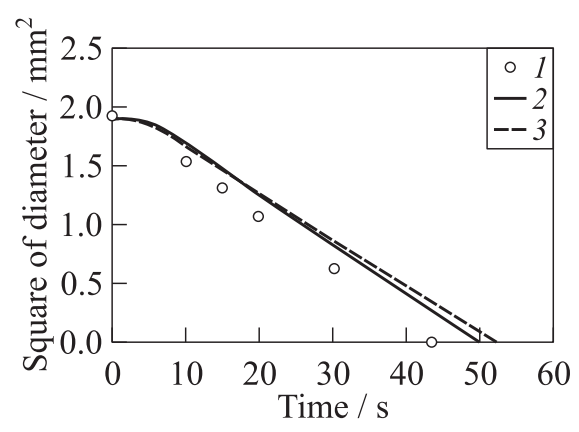

(a)

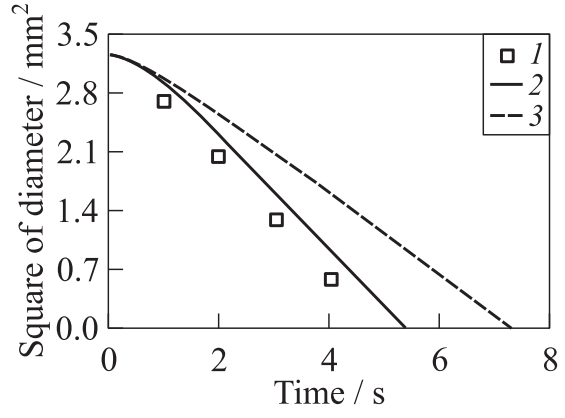

(b)

Figure 4 Distribution of square diameter of water $(a)$ and $n$-heptane $(b)$ : $1-$ experiments; 2 - equilibrium model; and 3 - nonequilibrium model 


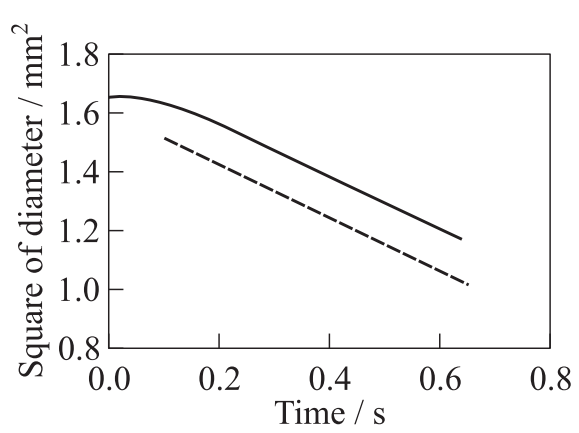

Figure 5 Distribution of square diameter of hydrazine

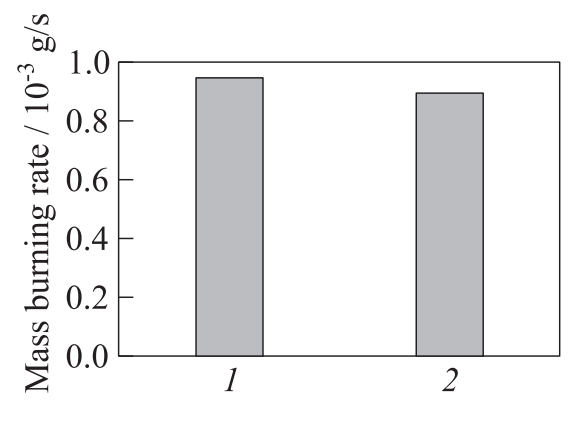

Figure 6 Comparison of mass burning rate: 1 - experiments; and 2 - numerical simulation

As shown in Fig. 4b, the numerical result which is calculated by the equilibrium model and the experimental are well corresponding each other, but the result of nonequilibrium model does not correspond to the experimental one. The nonequilibrium model evaluates a 45 percent longer evaporation time based on the equilibrium model than that of the equilibrium model. It can be considered that the reason why the nonequilibrium model evaluates such result unlike the case of water is involved in the difference of evaporation rate $(\dot{m})$ between water droplet and $n$-heptane droplet.

Equations (7) and (8) indicate that the bigger the evaporation rate, the greater is the difference from the equilibrium model. Therefore, the difference between the two models is significant in the case of $n$-heptane which has faster evaporation rate.

From Fig. 4, it can be confirmed that the numerical code used in this study with the equilibrium evaporation model has satisfactory accuracy for the problem of a single droplet evaporation.

Figure 5 shows time series of square of diameter of hydrazine droplet. The mass burning rate is calculated by means of Eq. (11) using the gradient of the linear part of the figure (dashed curve, $K=0.8724 \mathrm{~mm}^{2} / \mathrm{s} ; \dot{M}=0.8972 \mathrm{mg} / \mathrm{s}$ ). The equilibrium evaporation model is used in this simulation.

Figure 6 shows the mass burning rate of the experiment and of the simulation. As shown in the figure, both values are well corresponding to each other and the difference is about $6 \%$ based on the experiment. Therefore, it can be thought that the numerical code employed here has the sufficient precision for the evaporation problem of hydrazine droplet with chemical reaction. 


\section{SUMMARY}

First, the evaporation simulation without chemical reactions for single droplets of water and $n$-heptane are performed. The two evaporation models, such as the equilibrium and the nonequilibrium, are compared with existing experimental results, and it is confirmed that the equilibrium model shows sufficient accuracy. Next, the evaporation simulation with chemical reactions for single droplets of hydrazine are performed and the accuracy of the numerical code is confirmed by the same way. From these simulations, it is considered that the code has reasonable accuracy for droplet simulation with evaporation and combustion.

\section{REFERENCES}

1. Daimon, W., Y. Gotoh, and I. Kimura. 1991. Mechanism of explosion induced by contact of hypergolic liquids. J. Propul. Power 7(6):946-952.

2. Daimon, Y., H. Terashima, and M. Koshi. 2014. Chemical kinetics of hypergolic ignition in $\mathrm{N}_{2} \mathrm{H}_{4} / \mathrm{N}_{2} \mathrm{O}_{4}-\mathrm{NO}_{2}$ gas mixtures. J. Propul. Power 30(3):707-716.

3. Tani, H., H. Terashima, M. Kosh, and Y. Daimon. 2015. Hypergolic ignition and flame structures of hydrazine/nitrogen tetroxide co-flowing plane jets. Proc. Combust. Inst. 35(2):2199-2206.

4. Crowe, C. T., M. P. Sharma, and D. E. Stock. 1977. The particle-source-in cell (PSICell) model for gas-droplet flows. J. Fluid. Eng. — T. ASME 99(2):325-332.

5. Godsave, G. A. 1953. Studies of the combustion of drops in a fuel spray - the burning of single drops of fuel. 4th Symposium (International) on Combustion $4(1): 818-830$.

6. Bellan, J., and K. Harstad. 1987.Analysis of the convective evaporation of nondilute clusters of drops. Int. J. Heat Mass Tran. 30(1):125-136.

7. Miller, R., K. Harstad, and J. Bellan. 1998. Evaluation of equilibrium and nonequilibrium evaporation models for many-droplet gas-liquid flow simulations. Int. J. Multiphas. Flow 24(6):1025-1055.

8. Fujimoto, K., and K. Fujii. 2007. Study on the automated CFD analysis tools for conceptual design of space transportation vehicles. 5th Joint ASME/JSME Fluids Engineering Conference Proceedings. FEDSM 2007-37128.

9. Shima, E., and K. Kitamura. 2011. Parameter-free simple low-dissipation AUSMfamily scheme for all speeds. AIAA J. 49(8):1963-1709.

10. Morii, Y., H. Terashima, M. Koshi, T. Shimizu, and E. Shima. 2014. Fast and robust time integration method for stiff chemical kinetic ODEs. AIAA Paper No. 20143920. 
11. Kobayasi, K. 1954. A study on the evaporation and combustion of a single droplet: 1st report: Surface temperature of evaporating droplet and its measurement. T. Jpn. Soc. Mech. Eng. 20(100):831-837.

12. Hiroyasu, H., T. Kadota, and T. Senda. 1974. Evaporation of a single droplet at elevated pressures and temperatures: Part 1, Experimental study. T. Jpn. Soc. Mech. Eng. 40(339):3147-3154.

13. Allison, B. C. 1971. Hybrid and decomposition combustion of the hydrazine fuels. NASA CR-72977. 